\title{
Vacuum System Developments at the National Synchrotron Radiation Research Center -from the 1.5 GeV TLS to the $3.3 \mathrm{GeV}$ TPS*
}

\author{
June-Rong CHEN*1,2, Gao-Yu HSIUNG*1, Che-Kai CHAN*1, Tsai-Lieh YANG*1, \\ Chien-Kuang KUAN*1, Shen-Nung HSU*1, Chin-Chun CHANG*1, \\ Jia-Ying YANG*1, Hsin-Pai HSUEH*1 and Ching-Lung CHEN*1 \\ *1National Synchrotron Radiation Research Center, Hsinchu Science Park, Hsinchu 30076, Taiwan \\ *2Department of Biomedical Engineering \& Environmental Science, National Tsing-Hua University, Hsinchu 30043, Taiwan
}

(Received November 10, 2005, Accepted October 14, 2006)

This article describes the design and operation of the vacuum system of the $1.5 \mathrm{GeV}$ Taiwan Light Source (TLS) at the National Synchrotron Radiation Research Center (NSRRC). The TLS vacuum system has aluminum beam ducts, an oil-less fabrication process, a low impedance structure, an oil-less pumping system, little dust and an effective vacuum safety interlock system. Operating already for 12 years, TLS vacuum system has been highly reliable.

A low-emittance 3.3 GeV synchrotron light source, the Taiwan Photon Source (TPS, with a circumference of $518.4 \mathrm{~m}$ ), at the NSRRC is proposed to meet the requirements of advanced scientific study. Given the strictness of these requirements, the TPS vacuum system must be constructed to perform well in many areas, with a low vacuum pressure, little dust, a stable mechanical structure and a low risk of material failure caused by a high heat load or radiation damage. The vacuum system of the TPS has been preliminarily designed based on experience gained at the TLS. This work presents the technical design of the TPS vacuum chambers, the pumping configuration and some special components, emphasizing the considerations in obtaining an ultrahigh vacuum, the factors that influence beam quality and the reliability of the vacuum system.

\section{Introduction}

Many third-generation synchrotron light sources have been constructed and operated in recent years to investigate the frontiers of science. Of these, the $1.5 \mathrm{GeV}$ Taiwan Light Source (TLS) at the National Synchrotron Radiation Research Center (NSRRC), has been successfully operating for 12 years since its dedication in 1993 . Recently, another global wave of construction of synchrotron light sources in the energy range of $3 \mathrm{GeV}$ (intermediate energy) ${ }^{1)}$ has sought to satisfy the requirements of such rapidly growing fields as bio- and nanotechnologies. Accordingly, the NSRRC has begun to design a high-performance intermediate-energy synchrotron light source, called the Taiwan Photon Source (TPS).

The TLS is a machine with an electron beam energy of $1.5 \mathrm{GeV}$, a circumference of $120 \mathrm{~m}$, and six straight sections, each with a length of $6 \mathrm{~m}$. Table 1 presents the main parameters of the TLS and the TPS. Six insertion devices have been installed after the completion of the first phase of construction of the machine without an insertion device. One more elliptical polarized undulator and three superconducting wigglers are under construction and will be installed in two years. The stability and reliability of the machine have also been significantly improved over the last few years. However, the number of straight sections, the electron beam energy and the brightness of the photon beam are too small to meet the increasing demands of users.

* Presented as an invited talk in the 46th annual symposium of VSJ in 2005 .
Table 1 Main machine parameters of the TLS and the TPS.

\begin{tabular}{l|c|c}
\hline \multicolumn{1}{c|}{ Parameter } & TLS & TPS \\
\hline Lattice Energy (GeV) & 1.5 & $3.0-3.3$ \\
\hline Current (mA) & 200 & $400(300 @ 3.3 \mathrm{GeV})$ \\
\hline Circumference (m) & 120 & 518.4 \\
\hline No. of Long Straights & 6 & 24 \\
\hline Emittance (nm-rad) & 25 & 1.7 \\
\hline Bending Radius (m) & 3.495 & 7.257 \\
\hline Bending field (T) & 1.43 & 500 \\
\hline RF Frequency (MHz) & 500 & Proposing \\
\hline Operation Status & Operation & \\
\hline
\end{tabular}

The TPS has an electron beam energy of 3.0-3.3 GeV, a circumference of $518.4 \mathrm{~m}$ and 24 straight sections; it is 10,000 times as bright as the TLS in the photon energy range 1-20 keV. Fig. 1 plots curves of the photon beam brightness of the TPS, and of the TLS for comparison. The TPS will be constructed with high stability, low emittance, high brightness and high reliability. The following targets are challenging: electron beam emittance $<2 \mathrm{~nm}$-rad, electron beam orbit fluctuation $<0.2 \mu \mathrm{m}$, beam size fluctuation $<0.2 \mu \mathrm{m}$, photon beam intensity fluctuation $<0.1 \%$, photon beam brightness $>10^{21}$ $\mathrm{p} / \mathrm{s} / 0.1 \% \mathrm{bw} / \mathrm{mm}^{2} / \mathrm{mrad}^{2}$ (at $10 \mathrm{keV}$ ), injection efficiency $>90 \%$, machine up-time $>98 \%$ and a trip rate of less than once per week.

The vacuum system of the machine is critical in achieving the strict targets. The following sections 


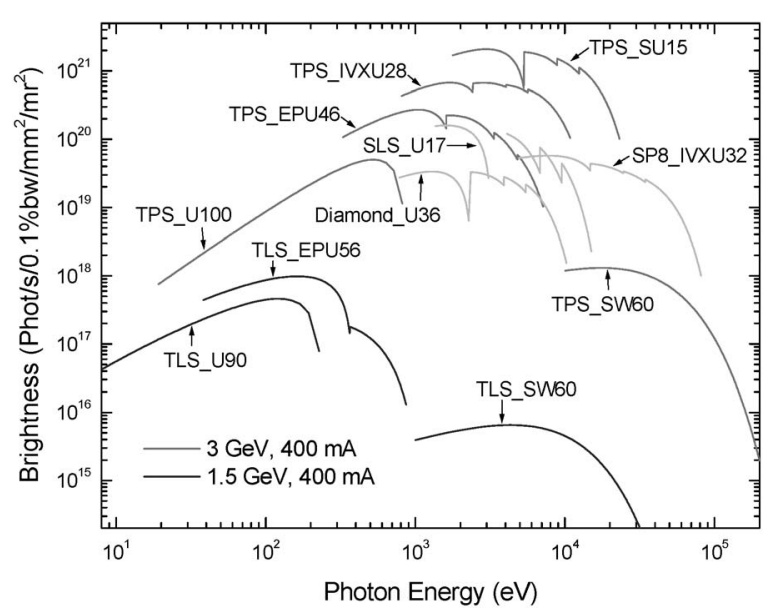

Fig. 1 Curves of the photon beam brightness of the TPS, and of the other light sources for comparison.

describe the experience gained from the TLS vacuum system and the design of the TPS vacuum system, emphasizing the considerations in obtaining an ultrahigh vacuum, the factors that influence beam quality and the reliability of the vacuum system.

\section{Vacuum Chambers and Treatments}

\section{A. TLS}

Aluminum has the advantages of high thermal conductivity, low outgassing rate, lightness, ease of extrusion, ease of design with simple structure (such as obtained using co-extruded or co-machined cooling channels) and ease of machining. Therefore, aluminum was selected as the material for making vacuum chambers of the TLS beam ducts. Many beam ducts have been developed for the TLS. The vacuum chambers can be classified into three categories -chambers for bending magnets (B-chamber), chambers for quadrupole and sextupole magnets (S-chamber) and chambers for insertion devices (ID-chamber). ${ }^{2-4)}$
The B-chambers were constructed using the oil-less numerical controlled (NC) machining process. Two pieces (lower and upper halves) of the aluminum plates were NC-machined according to computer-aided drawings. Ethyl alcohol was employed as the lubricant during machining. A thin and clean oxide layer is generated on the surface in the reaction between the fluid and the active fresh aluminum surface. Ethyl alcohol machining involves a thinner oxide layer than that of the other process, promoting the low outgassing rate. Degrease cleaning was performed on the aluminum pieces before welding to remove contaminants from the surface that are formed there in the transportation or some handling processes. The two halves of the B-chamber were welded by tungsten-inert-gas (TIG) welding in a clean room with controlled humidity $(<50 \% \mathrm{RH})$. The deformation was controlled to be $<0.3 \mathrm{~mm}$ in the B-chamber that was nearly 2 m long. ${ }^{2)}$

The S-chambers and the ID-chambers were made by extrusion. The straightness of the chambers was controlled to be $\sim 0.5 \mathrm{~mm} / \mathrm{m}$ following extrusion. Some chambers were further machined to a straightness of $<0.2 \mathrm{~mm} / \mathrm{m}$ to enable them to fit in the limited space of some superconducting IDs. Table 2 lists the specifications of several ID-chambers made for different insertion devices at the TLS.

The S- and ID-chambers were chemically cleaned (using $\mathrm{NaOH}, \mathrm{HF}+\mathrm{HNO}_{3}$ and de-ionized water ultrasonic cleaning $)^{5)}$ before TIG welding of other aluminum components to form a complete flange-to-flange chamber. The flanges and bellows in the beam ducts of the TLS are made mostly of aluminum. Aluminum gaskets, bolts and nuts are utilized to seal both aluminum-to-aluminum and aluminum-to-stainless steel flanges. The outgassing rate and the mechanical tolerance of the B-, S- and IDchambers satisfied the requirements of the TLS. All the steps in the fabrication of the chambers were successful, as evidenced by the performance of the operation of the electron beam.

Table 2 The specifications of the ID-chambers made for different insertion devices at the TLS.

\begin{tabular}{|c|c|c|c|c|c|c|c|}
\hline Name of Straight Section & $\mathrm{R} 1$ & $\mathrm{R} 2$ & R3 & R4 & R5 & R6 & R6 \\
\hline Name of Insertion Device & SWLS & EPU5.6 & U5 & SW6 & W20 & U9 & IASW6 \\
\hline Minimum Pole Gap (mm) & 55 & 18 & 18 & 18 & 22.5 & 18 & 19 \\
\hline Length of ID & 0.6 & 3.9 & 3.9 & 1.405 & 3.1 & 4.5 & 0.5 \\
\hline Length of ID Chamber(m) & 0.84 & 4.16 & 4.16 & 1.4 & 3.16 & 4.76 & 0.96 \\
\hline Inside Vert. Height (mm) & 20 & 13 & 13 & 11 & 17.5 & 13 & 12 \\
\hline Inside Hori. Width (mm) & 100 & \multicolumn{5}{|c|}{80} & 98 \\
\hline Flatness (mm) & $< \pm 0.2$ & $< \pm 0.2$ & $< \pm 0.2$ & $< \pm 0.2$ & $< \pm 0.2$ & $< \pm 0.2$ & $< \pm 0.2$ \\
\hline Cooling Channel & NA & Outboard & Outboard & NA & Both Sides & Outboard & NA \\
\hline Length of NEG Pump (m) & NA & 3.54 & 3.54 & NA & 2.62 & 4.14 & NA \\
\hline Time of Installation & April, 2002 & March, 1997 & March, 1997 & Dec., 2003 & March, 1995 & June, 1998 & Dec., 2005 \\
\hline
\end{tabular}




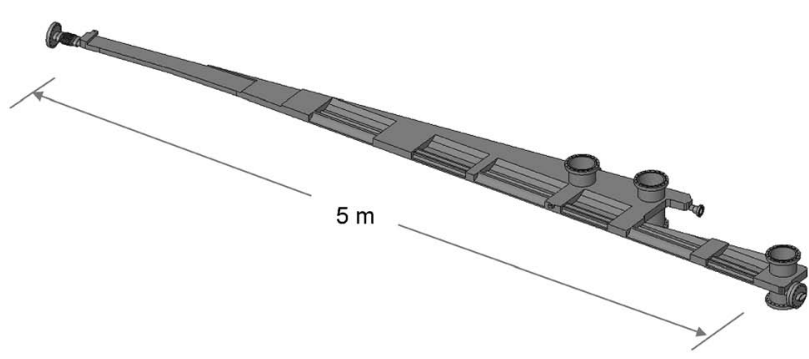

Fig. 2 A preliminary 3D schematics of the TPS B-chamber.

\section{B. TPS}

The technologies used to fabricate the TLS chamber will also be used to fabricate the TPS chamber. Fig. 2 depicts a preliminary design of the TPS B-chamber. The B-chamber will be fabricated by NC machining with ethyl alcohol as a lubricant to ensure that the surface of the chamber is clean enough. An automatic welding process will be developed for the large-size B-chamber, with an eye to manpower and budget. The S- and IDchambers of the TPS will be made by extrusion. If required, the chemical cleaning process of the TLS chamber will be modified or new processes will be used, to fulfill the requirement of environmental protection.

\section{Outgas and Pumping Configurations}

\section{A. TLS}

In a synchrotron light source, a large amount of photon-stimulated desorption (PSD) is caused when synchrotron light hits the chamber wall. Like the choice of the chamber material and the surface treatment method, an effective pumping configuration is crucial in reducing the vacuum pressure. Two pumping configurations, distributed pumping and localized pumping, are typically employed to reduce the average pressure in a conductance-limited system. At the TLS, the bending chamber was designed to confine most of the PSDs. A large pump was installed underneath the PSD location ${ }^{2}$, so the conductance between the gas source and the pump is less limited. However, distributed pumping with non-evaporable getter (NEG) strips was used in the ID-chambers ${ }^{4)}$. Some other lumped pumps were located where the pressure is high. A computer simulation was conducted before the design of the pumping configuration was finalized. An average pressure of $\sim 1 \times 10^{-7} \mathrm{~Pa}$, corresponding to a beam lifetime of 10 hours, was achieved at a desorption coefficient of $\eta=1 \times 10^{-5}$ molecules/electron. The operation results are consistent with the simulation results, except for slight deviations downstream in the long straight, where the cleaning is slower. $\left.{ }^{6}\right)$

\section{B. TPS}

Table 3 presents the PSD- related parameters of the TLS and TPS. Notably, the table reveals that the total PSD of the TPS is only about double that of the TLS (with the same beam current), although the circumference is 4.3 times larger, so the average pressure of the TPS could potentially be lower than that of the TLS, if a good pumping configuration is designed. The following designs support an average pressure of $\sim 3.3 \times 10^{-8} \mathrm{~Pa}$,
Table 3 The PSD- and thermo-related parameters of the TLS and TPS. (at a beam current of $400 \mathrm{~mA}$ and $\eta=1 \mathrm{E}-5 \mathrm{molec} . / \mathrm{e}$ )

\begin{tabular}{|c|c|c|}
\hline Parameter & TLS & TPS \\
\hline Critical Photon Energy (keV) & 2.14 & 8.25 \\
\hline Maximum Power Density at Crotch $\left(\mathrm{W} / \mathrm{mm}^{2}\right)$ & $\sim 4$ & 22 \\
\hline Total PSD at normal incident $\left(\mathrm{Pa}^{*} \mathrm{~m}^{3} / \mathrm{s}\right)$ & $1.58 \mathrm{E}-06$ & $3.2 \mathrm{E}-06$ \\
\hline No. of cells & 6 & 24 \\
\hline PSD per cell @ $400 \mathrm{~mA}\left(\mathrm{~Pa}^{*} \mathrm{~m}^{3} / \mathrm{s}\right)$ & $2.64 \mathrm{E}-07$ & $1.33 \mathrm{E}-07$ \\
\hline Nominal Pumping Speed (per cell) & $\sim 4000 \mathrm{~L} / \mathrm{s}$ & $\sim 4000 \mathrm{~L} / \mathrm{s}$ \\
\hline Pump ports per cell & 13 & 10 (off axis) \\
\hline Calculated Pressure Increase @ 400 mA (Pa) & $6.6 \mathrm{E}-08$ & $3.3 \mathrm{E}-08$ \\
\hline Operational Pressure Increase $(\mathrm{Pa})$ & $\left(\begin{array}{c}1 \mathrm{E}-07 \\
(@ 200 \mathrm{~mA})\end{array}\right.$ & NA \\
\hline Bending Angle of Dipole Magnet (degree) & 20 & 7.5 \\
\hline $\begin{array}{l}\text { Photon span of the synchrotron light inside } \\
\text { the B-chamber (degree) }\end{array}$ & 15.4 & 6.96 \\
\hline $\begin{array}{l}\text { Percentage of the synchrotron light inside } \\
\text { the B-chamber }\end{array}$ & $77 \%$ & $92.8 \%$ \\
\hline Incident angle at straight-chamber (degree) & $4.61-0.22$ & $0.54-0.11$ \\
\hline $\begin{array}{l}\text { Multiplication factor in desorption yield, } \\
\mathrm{F}(\theta) \text {, for small angle incident }\end{array}$ & $3.52-7.5$ & 7.5 \\
\hline
\end{tabular}

even at a high desorption rate of $1 \times 10^{-5}$ molecules/electron.

The ante-chamber and localized pumping configuration in the TPS B-chambers are designed. Fig. 2 schematically depicts the TPS B-chamber. The length of the B-chamber varies from 4 to $5 \mathrm{~m}$, according to the lattice structure of the accelerator. The incident angle of the photon beam on the wall is $\sim 90$ degree in the B-chamber, to minimize the photoelectric yield, and thus the gas desoption yield. The percentage of the synchrotron light that is distributed inside the bending chamber is approximately $93 \%$ in the TPS ( $77 \%$ in the TLS). It is more critical to design the pumps in the B-chamber of the TPS than the TLS. Because the B-chamber of the TPS is larger than that of the TLS, three to four pumps can be installed in the TPS B-chamber and more design options can be implemented to reduce the local pressure.

In the straight section, although the photon flux is markedly less than that in the B-chamber, the PSDs can not be ignored because the incident angle of the photon beam on the wall of the chamber is smaller, and thus a higher desorption yield. Two different designs are considered to reduce the average pressure in the straight chamber. The distributed pumping with NEG strips is utilized in the ID-chambers, where the conductance and the space for the pumps are extremely limited. The localized pumping approach is applied in the long straight section, such as the injection section, which constrains the space for the pumps less than the other straight sections. 




(a)

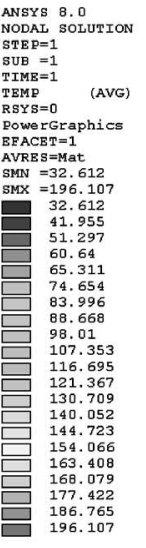



(b)

Fig. 3 Before (a) and after (b) using stepped surfaces and fins in the cooling channel enables the maximum temperature of the chamber surface to be reduced from $\sim 196^{\circ} \mathrm{C}$ to $\sim 109^{\circ} \mathrm{C}$.

\section{Thermal and Mechanical Issues}

\section{A. TLS}

The low electron beam energy and the high thermal conductivity of the aluminum chamber are such that the thermal density of the synchrotron light that hits the chamber wall and the photon absorbers is not a problem at TLS. The thermal density on the B-chamber is $\sim 4$ $\mathrm{W} / \mathrm{mm}^{2}$. However, thermal variations caused by the decay of the beam current and the variations in the temperature of the cooling water did lead to some problems in beam stability. The fluctuation of the temperature of the cooling water has been improved from $\pm 1^{\circ} \mathrm{C}$ in the early commissioning stage to $\pm 0.1^{\circ} \mathrm{C}$ currently. The motion of some important components, including magnets and beam position monitors, that link to or touch the vacuum chamber, directly or indirectly through the magnet girder, was reduced accordingly.

\section{B. TPS}

The thermal power density of the synchrotron light on the B-chamber of the TPS will be unacceptably high if the chamber wall is located at the same distance from the source point as the TLS. Increasing the distance is an effective means of reducing the thermal load on the chamber wall. A larger B-chamber not only corresponds to a lower pressure, as stated in the preceding section, but also a reduction in the thermal density problem at the TPS. The maximum power density of the synchrotron light that hits the chamber wall is $\sim 22 \mathrm{~W} / \mathrm{mm}^{2}(400$ $\mathrm{mA}$ at $3 \mathrm{GeV}$ ), for the B-chamber as designed herein. Further improving the geometry of the cooling channel and the shining synchrotron light onto the stepped surface $(0.4 \mathrm{~mm}$ vertical $\times 2 \mathrm{~mm}$ horizontal, Fig. 3), enabled the maximum temperature of the chamber surface to be maintained at $\sim 109^{\circ} \mathrm{C}\left(\sim 138^{\circ} \mathrm{C}\right.$, at the maximum capacity $350 \mathrm{~mA}$ at $3.3 \mathrm{GeV}$ ), which is tolerable for aluminum.

The support of the vacuum chamber will be directly fixed to the ground, and so is isolated from the girder of magnets, to eliminate the problems of mechanical dis- placement and vibration that occurred in the TLS. ${ }^{7)}$

\section{Installation and Reliability}

\section{A. TLS}

Not only gas molecules but also micro dusts in the vacuum system of the synchrotron light source are of concern. Micro dust in the beam duct can be excited and ionized by scattered light or electrons. The ionized micro dust can be trapped in the potential well formed by the circulating beam. The collision of the circulating beam with the micro dust causes an abrupt drop in the beam current. All welding and pre-assembly processes were undertaken in clean rooms to keep the contamination away from the micro dusts. Clean booths were employed during the installation in the storage ring tunnel.

Like the installation of the chambers and components, the generation and transportation of micro dusts in the vacuum must also be dealt with. Micro dust can also be generated in a vacuum, including in an ion pump and from getter materials. In the TLS vacuum system, ion pumps were operated only after baking at a pressure of $10^{-6} \mathrm{~Pa}$ or lower. The high voltage supplied to ion pump was kept at a low value of $\sim 3 \mathrm{kV}$ to reduce the rate of formation of micro dust. During venting, the venting rate was maintained at a reasonable low value to prevent the stirring up of the micro dusts.

In-situ baking is used in the TLS vacuum system. Tape heaters are utilized to bake the chamber at up to $150^{\circ} \mathrm{C}$ for aluminum parts, and up to $200-250^{\circ} \mathrm{C}$ for stainless steel parts. All the gauges, ion pumps and NEGs are degassed or regenerated at the end of the baking process.

\section{B. TPS}

The procedure for handling micro dust in the TLS will be implemented in the TPS vacuum system. The baking process employed in the TPS will be modified, for reasons of manpower and available time. The chambers will be pre-baked in the laboratory and then vacuum-sealed for transportation.

A humidity prevention procedure will be implemented in installation, so the in-situ bake out process can be 
omitted. However, a baking system, using either hot water or tape heaters, will still be installed as a backup system that operates when the vacuum in the storage ring is broken.

A vacuum safety protection system is necessary in the TPS to protect the vacuum in a section from vacuum failure in another section. A multilayered and redundant protection system, like that used in the TLS, will be designed. ${ }^{8)}$ In each of the important devices, including ion pump (IP), ionization gauge (IG) and turbo-molecular pump (TMP), a self protection or alarm circuit is installed in the device controller. Relay-controlled pneumatic actuated valves will isolate the vacuum system in each section. Redundant sensors, such as the three IGs in a section, will be designed to reduce the probability of a false alarm. A reliable programmable logic controller will be programmed to perform the vacuum interlock processes. Like that of the main vacuum components of the safety interlock, the reliability of the supply of the compressed air and the cooling water will be continuously monitored. Thermal sensors will be distributed along all of the vacuum chambers to protect against the thermal problem.

\section{Beam Operation}

\section{A. TLS}

The accumulated beam dose of the TLS was $8622 \mathrm{Ah}$ in Nov. 2005. Fig. 4 plots the historical statistics of $\mathrm{P} / \mathrm{I}$ value (pressure increase per unit beam current) and $\mathrm{I} \tau$ value (the product of the beam current and the beam lifetime) against beam dose. The maximum $\mathrm{I} \tau$ value was $\sim 5567 \mathrm{mAh}$, so the beam lifetime was $\sim 27.5 \mathrm{~h}$ at a beam current of $200 \mathrm{~mA}$, which was the normal beam current of the TLS. Fig. 4 reveals that each installation of a new insertion device considerably increased $\mathrm{P} / \mathrm{I}$. Notably also, the I $\tau$ value vs. beam dose, shows that the increase in $\mathrm{I} \tau$ slows down when the beam dose exceeds $\sim 200 \mathrm{Ah}$. It implies that the beam lifetime is dominated by another factor than the gas pressure after a beam dose of $\sim 200$ Ah.

The TLS has been operating for 12 years. Annually, around 100 hour $(\sim 2 \%)$ of users' time is lost. Under $2 \%$ of failures are caused by failures of the vacuum system, representing under 2 hours in a year. The most common vacuum failures involve utility-related, such as compressed air and cooling water, components.

\section{B. TPS}

One characteristic of a third-generation synchrotron light source is the set of insertion devices (ID). Most IDchambers are long and narrow, so their conductance is small. The vacuum of the storage ring is lost during ID installation, so the vacuum pressure is severely degraded (as shown in Fig. 4). The gap is narrow so the impedance becomes more severe as the beam is operating. In a superconducting ID, the narrow gap of the ID-chamber can cause the problematic heating of the low-temperature components.

Designing an ID-chamber with effective pumping is important, especially for a machine with a very small emittance. Based on experience gained from the TLS,
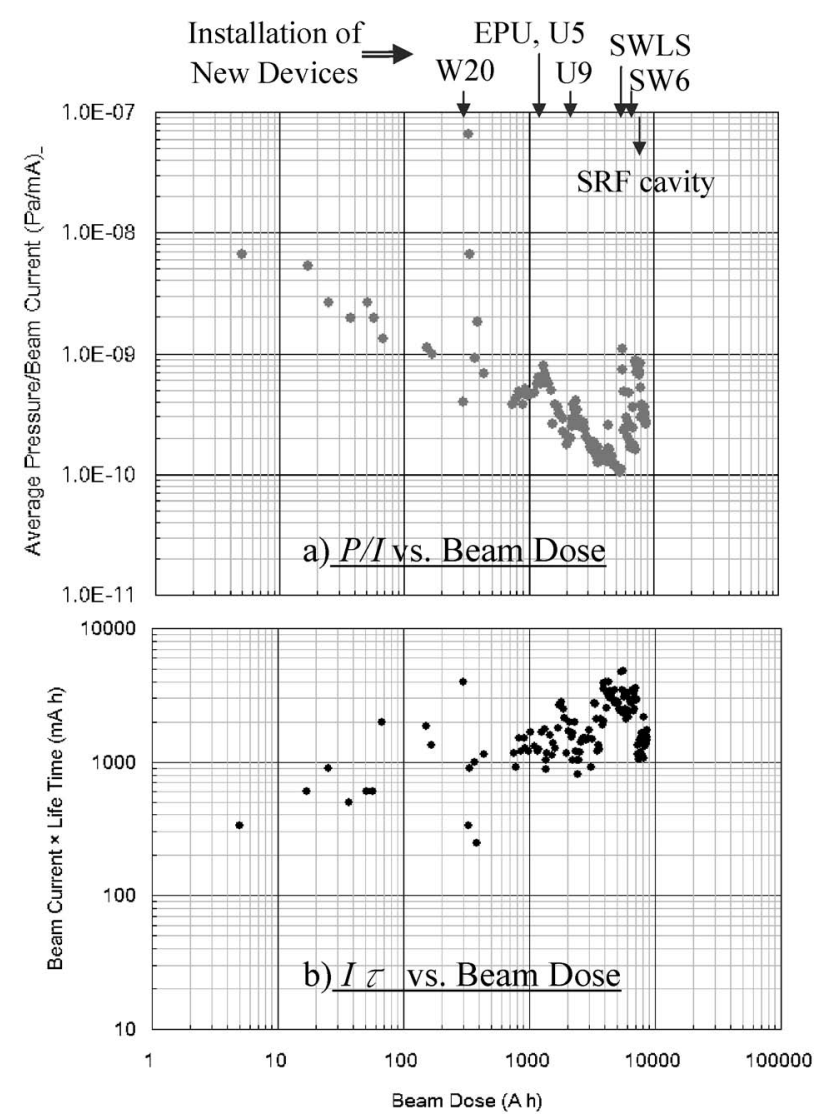

Fig. 4 The P/I value (a) and I $\tau$ value (b) of the TLS as a function of beam dose.

some issues are considered in designing the ID-chambers of the TPS. First, the vacuum pressure in the ID-chamber must be low, approximately $10^{-8} \mathrm{~Pa}$. The beam channel is too narrow to be coated with NEG material, so the NEG strip is the most appropriate in the distributed pumping in the ID-chamber. ${ }^{4)}$ However, some other pumps are required to remove the inert gases and methane, which the NEG cannot do. The magnetic field of the ID and the magnetic material contained in the NEG cause the NEG strip to be offset from the orbit of the beam, in a side-channel along the beam duct. This arrangement also reduces the potential effect of the drop off of the NEG powders in the beam channel. Second, most of the ID-chambers are to be fabricated and installed before the TPS is commissioned, to prevent the vacuum from being frequently broken and the allowing the beam dose on the ID-chamber to be accumulated effectively. The beam dose accumulates in the ID-chamber more slowly than in the B-chamber, so the ID-chamber must be cleaned continuously. Some ID-chambers will be unavailable at the commissioning of the TPS, and these will be helpfully be cleaned in a photon beam line before installation.

\section{Summary}

Aluminum vacuum chambers, fabricated by an oil-less process, and having a low impedance structure, an oilless and effective pumping system, an effective vacuum safety interlock system and a low-dust treatment and in- 
stallation procedure, were implemented in the TLS vacuum system. The 12 years of operating results of the TLS indicate that its vacuum system performs well in terms of both vacuum pressure and system reliability.

The vacuum system in the TPS must satisfy a higher standard than the TLS to meet the strict requirements of beam quality in this advanced low-emittance machine. The TPS vacuum system is preliminarily designed. Aluminum is selected as the chamber material, and the Bchamber is fabricated using an oil-less NC machining process. The S-chamber and ID-chamber are extruded; an appropriate chemical cleaning process will be employed in these chambers. The localized pumping and distributed pumping configurations are used in the Bchamber and the ID-chamber, respectively. The serious thermal problem is reduced by moving the crotch absorber in the B-chamber as far away from the source point as possible. The criteria are met by a larger Bchamber that is $\sim 5 \mathrm{~m}$ long. With respect to mechanical structure, the support for the vacuum chamber is independent of the magnet girder so that no force is transferred from the chamber to the magnet. Special care is taken in designing the ID-chambers. The pumps in the ID-chamber will be high-speed pumps and have little impact on the beam operation with high quality. Most of the ID-chambers are to be installed before the TPS is commissioned, to prevent frequent breaking of the vacuum and maintain better conditions in the ID-chamber to enable the performance of the machine to be continuously improved.

\section{Acknowledgement}

The authors would like to thank their colleagues in the NSRRC for providing some information for this article.

\section{Reference}

1) The papers in the proceedings of Shanghai Symposium on Intermediate-Energy Light Sources, the $25^{\text {th }}$ ICFA Advanced Beam Dynamics Workshop, Shanghai, Sep. 24-26, 2001.

2) J. R. Chen, G. S. Chen, D. J. Wang, G. Y. Hsiung and Y. C. Liu: Vacuum 41(7-9), 2079-2081 (1990).

3) J. R. Chen and Y. C. Liu: Vacuum 44(5-7), 545-548 (1993).

4) G. Y. Hsiung, D. J. Wang, J. G. Shyy, S. N. Hsu, K. M. Hsiao, M. C. Lin and J. R. Chen: J. Vac. Sci. \& Technol. A15(3), 723-727 (1997).

5) A. G. Mathewson, J. P. Bacher, K. Booth, R. S. Calder, G. Dominichini, A. Grillot, N. Hilleret, D. Latorre, F. Le Normandand and W. Unterlerchner: J. Vac. Sci. Technol. A 7(1), 77 (1989).

6) Y. C. Liu, J. R. Chen, G. Y. Hsiung and J. R. Huang: J. of the Vacuum Society of Japan 37(9), 751-757 (1994).

7) J. R. Chen, D. J. Wang, C. K. Kuan, Z. D. Tsai, D. S. Lee and H. C. Ho: Proceedings of Shanghai Symposium on Intermediate Energy Light Source (SSIELS-2001), Shanghai, Sep. 24-26, 2001. pp. 77.

8) J. R. Chen, T. S. Ueng, G. Y. Hsiung, Y. J. Hsu, J. G. Shyy, T. F. Lin and Y. C. Liu: J. Vac. Soc. of R.O.C., 11(1), 6-13 (1998). 